\title{
Exploring the Effect of Motion Type and Emotions on the Perception of Gender in Virtual Humans
}

\author{
KATJA ZIBREK, LUDOVIC HOYET, KERSTIN RUHLAND, and RACHEL MCDONNELL, \\ Graphics, Vision and Visualisation Group, Trinity College Dublin
}

\begin{abstract}
In this article, we investigate the perception of gender from the motion of virtual humans under different emotional conditions and explore the effect of emotional bias on gender perception (e.g., anger being attributed to males more than females). As motion types can present different levels of physiological cues, we also explore how two types of motion (walking and conversations) are affected by emotional bias. Walking typically displays more physiological cues about gender (e.g., hip sway) and therefore is expected to be less affected by emotional bias. To investigate these effects, we used a corpus of captured facial and body motions from four male and four female actors, performing basic emotions through conversation and walk. We expected that the appearance of the model would also influence gender perception; therefore, we displayed both male and female motions on two virtual models of different sex. Two experiments were then conducted to assess gender judgments from these motions. In both experiments, participants were asked to rate how male or female they considered the motions to be under different emotional states, then classified the emotions to determine how accurately they were portrayed by actors. Overall, both experiments showed that gender ratings were affected by the displayed emotion. However, we found that conversations were influenced by gender stereotypes to a greater extent than walking motions. This was particularly true for anger, which was perceived as male on both male and female motions, and sadness, which was perceived as less male when portrayed by male actors. We also found a slight effect of the model when observing gender on different types of virtual models. These results have implications for the design and animation of virtual humans.
\end{abstract}

Categories and Subject Descriptors: I.3.7 [Computer Graphics]: Three Dimensional Graphics and Realism—Animation

General Terms: Experimentation, Human Factors

Additional Key Words and Phrases: Facial animation, emotions, gender

ACM Reference Format:

Katja Zibrek, Ludovic Hoyet, Kerstin Ruhland, and Rachel McDonnell. 2015. Exploring the effect of motion type and emotions on the perception of gender in virtual humans. ACM Trans. Appl. Percept. 12, 3, Article 11 (July 2015), 20 pages.

DOI: http://dx.doi.org/10.1145/2767130

\section{INTRODUCTION}

Computer-generated virtual humans are an important part of many 3D graphical applications, and they are often used to invoke empathic responses from human users. Depending on the type of media and its technical limitations, some virtual humans are created to resemble human form and behavior

This work was sponsored by Science Foundation Ireland as part of the Cartoon Motion (303.G20586) and Captavatar (10/IN.1/13003) projects.

Authors' addresses: K. Zibrek, K. Ruhland, and R. McDonnell, Graphics, Vision and Visualisation Group, Trinity College Dublin, College Green, Dublin 2, Ireland; emails: \{zibrekka, ruhlandk\}@tcd.ie, Rachel.McDonnell@scss.tcd.ie; L. Hoyet, INRIA Rennes - Bretagne Atlantique Centre, 35042 Rennes Cedex, France; email: ludovic.hoyet@inria.fr.

Permission to make digital or hard copies of part or all of this work for personal or classroom use is granted without fee provided that copies are not made or distributed for profit or commercial advantage and that copies show this notice on the first page or initial screen of a display along with the full citation. Copyrights for components of this work owned by others than ACM must be honored. Abstracting with credit is permitted. To copy otherwise, to republish, to post on servers, to redistribute to lists, or to use any component of this work in other works requires prior specific permission and/or a fee. Permissions may be requested from Publications Dept., ACM, Inc., 2 Penn Plaza, Suite 701, New York, NY 10121-0701 USA, fax +1 (212) 869-0481, or permissions@acm.org.

(c) 2015 ACM 1544-3558/2015/07-ART11 $\$ 15.00$

DOI: http://dx.doi.org/10.1145/2767130 
as much as possible. To capture the natural subtleties of human motion, motion capture technology is often used to record the motion of real actors. The motion is then applied to virtual characters, possibly of different appearance, shape, and even sex. To create believable virtual humans, we need to know how these elements interact with each other, as some combinations might produce undesired effectsfor example, the viewer might recognize that a female motion is applied to a male character. Having a better understanding of these interactions not only would aid the design of believable virtual humans but also would provide a better understanding of human perception in general.

The creation of believable virtual characters involves the transmission of correct visual information to the viewer. In the literature, different factors have been studied, including how sex and emotions are perceived from the motion of virtual characters, which we further explore in this article. The implications of sex recognition from motion originates from the work of Kozlowski and Cutting [1977], who found that the sex of walkers could be identified from point-light displays (lights attached to the major joints in the body). Their finding might prevent us from applying motion from an actor whose sex does not match the sex of the virtual model, as was shown by previous studies (e.g., McDonnell et al. [2009]). Many studies have also explored the perception of emotion expressed from body and facial motion, finding that basic emotions can be identified with a certain level of accuracy (e.g., see Bassili [1978], Atkinson et al. [2004], and Crane and Gross [2007]). Considering that humans have a high sensitivity to emotions conveyed by other humans, the transmission of correctly identified emotions is highly important to avoid ambiguity in perception of virtual humans. Therefore, the results of the mentioned studies focusing on emotion perception from motion cues have a high applicable value.

However, studies from psychology have demonstrated that gender differences in emotion are accounted for in terms of social and cultural context as a result of gender-stereotypic socialization. For instance, stereotypes of people living in Western societies usually describe women as more affiliative, and more likely to show happiness, and men as being more dominant, and therefore more likely to show anger [Hess et al. 2004; Fischer et al. 2004; Plant et al. 2000]. Because of this attribution bias, the perception of gender could be affected by the presented emotion. A study confirmed this by demonstrating that there is an interaction between perceived sex from body motions and emotions expressed from throwing motions [Johnson et al. 2011]. By using point-light displays, they demonstrated that angry throwing motions are overwhelmingly perceived as male and sad motions as female, regardless of the actual sex of the thrower. In this article, we further explore how sex and emotions are perceived from human motions and hypothesize that emotions affect sex perception when other cues for sex are less apparent. For the purposes of our study, we chose to test the effect of emotion on gender judgments on two different types of motion: walking and conversation. Walking motions have been shown to exhibit gender information through hip sways and shoulder movements [Johnson and Tassinary 2005]; therefore, we expect that gender judgments would be less affected by the portrayed emotions. On the other hand, since conversational motions carry less physiological cues and are mostly a product of socialization and cultural differences, we expect them to be more affected by gender stereotypes that exist around emotions. Therefore, our main interest in this work is how different motion types influence gender perception in different emotional conditions (our use of the terms gender and sex is defined in Section 4.1.1).

Appearance is another element that influences the perception of sex. The motion of virtual models with exaggerated female features appear more female, and the same effect can be found on male models with exaggerated male characteristics [McDonnell et al. 2009]. It would thus be reasonable to take into account the appearance of the model when constructing a character of a certain sex. For this reason, we use virtual models of both sexes to display motions, which is an important distinction from other studies that have used only one model or point-light displays. We thus provide practical implications for character creation. 


\section{BACKGROUND}

Since the early work of Johansson [1973] on the perception of human motion from point-light displays, it has been shown that humans are extremely capable of recognizing and categorizing human motion even with very little information. Several studies focused on understanding how gender is expressed through human motion. For instance, it has been shown that gender can be perceived through body motion alone (e.g., Kozlowski and Cutting [1977]), with hip sway often indicating female motion and shoulder movement indicating male motion [Johnson and Tassinary 2005]. A more recent study revealed an effect of motion and body shape on the perceived sex of walking virtual characters [McDonnell et al. 2009], where exaggerated male or female body shape influenced sex perception. Other studies also focused on the recognition of gender from facial motion, using either androgynous faces [Hill and Johnston 2001; Morrison et al. 2007] or comparisons with point-light displays [Hill et al. 2003]. These studies found that gender can be recognized from facial motion only, but that specific cues, such as excessive nodding, blinking, and overall amount of movement, play an important role in recognizing the female sex [Morrison et al. 2007]. Similarly, Hill et al. [2001] showed that head motion alone is less useful for discriminating sex than facial movements.

Considering that humans have a high sensitivity to emotions conveyed by other humans, researchers also have investigated how emotions are perceived and recognized in human facial expressions, body poses, and motions. The studies usually have investigated six basic emotions: anger, happiness, sadness, fear, surprise, and disgust, according to the classification of Ekman [1992]. Studies using pointlight displays for recognition of movement show that emotions can be identified from either body motion (e.g., for full body motions, see Atkinson et al. [2004] and Crane and Gross [2007]; for upper body motions, see Volkova et al. [2014]; for arm motions, see Pollick et al. [2001]; and for facial motion, see Bassili [1978]). Similar results have been found on motion applied to virtual characters [Ennis et al. 2013]. Additionally, recognition rates are improved when body and facial motions are combined [Clavel et al. 2009], showing that both facial and body cues have a specific role in conveying emotion. Research also has shown that there are particular areas of the face [Schyns et al. 2009] and certain body motions [De Meijer 1989] that convey most of the information about the portrayed emotions-for example, corners of the mouth are important in recognizing happiness, which suggests that the recognition of emotion can be improved when this behavioral data is present.

Gender stereotypes impact both the production and perception of emotions [Brewer 1988]. Many gender studies done with subjects living in Western societies have shown that emotions are perceived to be gender specific [Hess et al. 2004; Fischer et al. 2004; Plant et al. 2000]. Overall, in these societies, women are believed to express more emotions than men, but some emotions are more male specific, such as anger, contempt, and pride [Plant et al. 2000]. It is also believed that females express more emotions that signal powerlessness, such as sadness and fear [Fischer et al. 2004]. Studies conducted on static images [Hess et al. 2004] also found differences in the way in which the same emotional expressions are perceived on female and male static faces. This is particularly the case for happiness and anger. While expressing anger, women were rated as more angry than men, and in the happy condition, men were rated as happier than women. Hess et al. [2004] attributed this finding to the contrast effect, as a woman showing anger or a man expressing happiness might violate the viewers expectation of gender stereotype and therefore would intensify the perceived emotional expression. Using video references of female and male actors portraying emotions, Battocchi et al. [2005] found that emotions are better recognized on female actors overall, especially anger and sadness. Although both studies explored the interaction between gender and emotion, neither separated appearance of the model from dynamic motion. They used either video references, where the sex of the actor was apparent, or static images and thus left out the component of dynamic motion. The study of Johnson 
et al. [2011] combined both aspects by exploring sex recognition bias on the perception of throwing a ball under different emotions using point-light displays. They found that an angry throw is perceived as more male and a sad throw as more female, which confirms previous findings.

According to the research that we have presented, sex and emotions can be recognized from motion, and there is evidence to support the fact that gender stereotypes for emotional expressions influence the perception of sex. However, none of these studies explored if this is true for different types of motion, especially considering that some motions, in the absence of emotional expressions, might carry more gender information than others, which could make them less prone to emotional bias. Drawing from the studies of walking motions that found that specific cues convey sex information [Cutting 1978], we assumed that walking motions would not be highly affected by emotional bias. Other relevant research on sex recognition from conversational motion, focusing on facial motion [Hill and Johnston 2001; Hill et al. 2003] showed above-chance recognition rates for both sexes (ranging from 58\% to $62 \%$ ). However, recognition rates from walking motions were generally found to be higher (around $70 \%$ [Kozlowski and Cutting 1977]) and mainly due to the information coming from shoulder and hip motion. We therefore expect that differences in sex recognition rates for conversation and walking will be present due to different amounts of physiological cues present in these two motions.

To test our hypothesis, we compare gender ratings for walking and conversational motions and expect the ratings for conversational motions to less accurately match the actual actor's sex. We also expect emotions to have an effect on how gender is perceived from conversational motion. Furthermore, since we expect our conversational characters to exhibit less gender cues than walking characters, we hypothesize that emotions expressed through conversation would affect the heuristic of sex judgments to a greater extent than they would for walking motions.

Finally, the research mentioned previously that investigated emotion and/or sex recognition used point-light displays or one virtual model to display the motions, whereas other previous work has shown that the choice of virtual model affects the perception of both motion [Chaminade et al. 2007] and sex of the virtual character [McDonnell et al. 2009]. For this reason, we used two models-one male and one female - to display the motions of both male and female actors.

To test our assumptions, we conducted two experiments. In Experiment 1 (Section 4), we studied gender perception for basic emotions expressed while walking, where we expected that gender judgments would not be highly affected by the displayed emotion. Experiment 2 (Section 5) was designed to test the same assumptions but for conversational motions, where we expected that gender stereotypes around specific emotions would influence the perception of gender to a greater extent than in the previous experiment. We conclude by comparing and discussing the results of both experiments in Section 6.

\section{STIMULI CREATION}

To address the question of gender and emotion perception and interaction on virtual characters, we chose to use natural motions captured on real actors to capture the subtleties and timings of real movements. The motion capture data used for both experiments in this article was acquired using a 21-camera Vicon optical system, where 52 markers were placed on the body. The motion capture data was taken from four female and four male actors. Two virtual characters of different sex were used to display the motions of the actors. We used congruent (where character sex matched actor sex) and incongruent (where character sex did not match actor sex) stimuli (see Figure 3). Other details of stimuli creation are discussed separately for Experiments 1 and 2.

For Experiment 1, actors were instructed to portray emotions through their walk. We did not capture face or finger motion. The captured body markers were used to compute the joint angle animations and mapped onto the virtual characters in Autodesk 3ds Max 2014. We created walking characters, 
displayed in central, full-body view and walking toward the camera. The faces of the virtual models were blurred out to direct participants' attention toward the body motion. In addition, static facial expressions might influence the overall emotional display of the walk. In total, 80 video clips of 3 seconds each were generated: 2 Models (Woman, Man) $\times 8$ Actors $(4 \mathrm{~F}, 4 \mathrm{M}) \times 5$ Emotions (Angry, Fear, Happy, Sad, and Neutral). Movies were created at 30 frames per second and at $1240 \times 900$ resolution and displayed on a 24-inch LCD screen.

For Experiment 2, we considered facial motion to be extremely important for recognition of conversational emotions, and therefore we added 36 markers on the face to capture facial motion alongside body motion. The actors were asked to remain stationary in the middle of the recorded capture space. The basic emotions were conveyed through acting out sentences that we selected from a validated list of affective sentences for spoken emotion identification [Ben-David et al. 2011], such as "Get out of my room!" for anger or "It's a beautiful day outside!" for happiness. From this validated list, we chose three sentences for each emotion (including Neutral). Since we wanted to examine the effect of motion alone, the voices of the actors, although captured, were not used in this experiment. No eye or finger motion was captured. As before, the body markers were used to compute the joint angle animations and then applied to virtual characters. The facial motion was exported as 3D marker motion, and a bone-based approach that used linear blend skinning was used to drive the facial geometry. To retarget the motion from actor to model, the markers were aligned to the head and then automatically adjusted to the position of the bones of the face of the character. The characters' facial bones were then constrained to their corresponding optical markers to produce the animation. We generated three clips for each emotional expression, as different sentences offer more variety in their interpretation. In total, 240 clips of 3 seconds each were generated: 2 Models (Woman, Man) $\times 8$ Actors $(4 \mathrm{~F}, 4 \mathrm{M}) \times 5$ Emotions (Angry, Fear, Happy, Sad, and Neutral) $\times 3$ Sentences. The character was displayed in the center of the screen, facing forward at the beginning of each clip. We selected a medium close-up view, as we wanted to provide information from both the facial movements and upper body of the actors while the legs were stationary. Movies were created at 30 frames per second and at $1240 \times 900$ resolution and displayed on a 24-inch LCD screen.

\section{EXPERIMENT 1}

In this experiment, we were interested in evaluating to what extent emotions would influence how observers perceive the gender of walking actors. Considering that evaluating the influence of emotions on gender perception judgments depends on accurate portrayal of emotions by actors (and therefore the accurate recognition by viewers), we also included an emotion recognition task in this experiment to ensure that the expected emotions were recognized above chance level through the walk.

\subsection{Experiment Design}

The experiment was composed of 24 participants (15M, 9F; average age: $24 \pm 4.5$ years). They were recruited mainly from the university, had normal or corrected-to-normal vision, and were all naïve to the purpose of the experiment. In addition, University ethical approval was granted for the experiment, and participants received a $€ 5$ book voucher to compensate for their time.

Participants were asked to read and sign the informed consent and then read through the information sheet, where they were familiarized with the details of the experiment. Afterward, they were seated in front of a computer screen and asked to follow the instructions displayed before them. The experiment was divided into two blocks: Gender Rating and Emotion Recognition. In each block, participants saw one model with all motions applied first, followed by the same motions on the other model. We randomized whether participants viewed the Man or the Woman model first. Clips were randomized within each block to avoid ordering effects. Participants saw 80 animations in each block: 


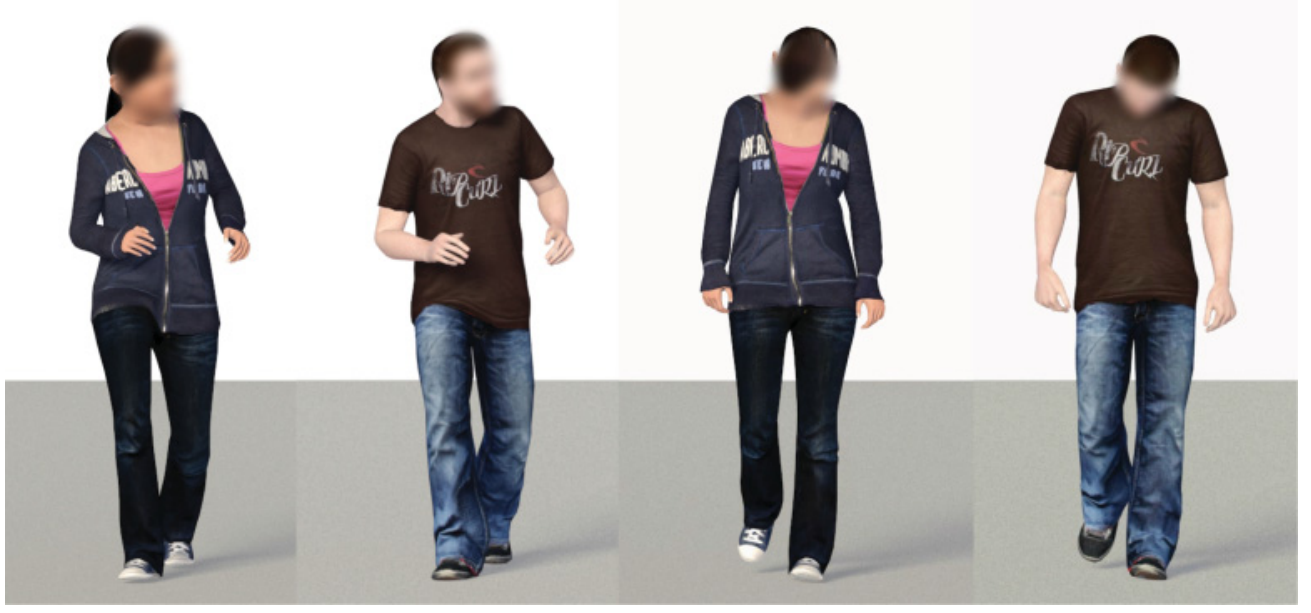

Fig. 1. Example of stimuli for Experiment 1. A Woman and a Man model with male actor's motion, portraying Fear (left), and female actor's motion, portraying Sad (right) applied.

2 Models (Woman, Man) $\times 8$ Actors (4F, 4M) $\times 5$ Emotions (Angry, Fear, Happy, Sad, and Neutral). An example of stimuli is shown in Figure 1.

4.1.1 Gender Rating. In the first block, participants were asked to rate the gender of the character based on motion cues. They were informed that the displayed motion would not necessarily match the sex of the model. After each clip, they rated Gender on a 5-point Likert scale from 1 (Very Male) to 5 (Very Female) using the number keys on a keyboard.

We decided to use the term gender for the rating task and the term sex for the actual underlying motion (male, female) based on the distinction between gender as a sociocultural construct associated with maleness and femaleness and sex as a demographic category [Unger 1979]. Because we instructed the participants to pass judgment on a 5-point scale of how female or male the motion was, and did not ask to categorize the motion as either male or female, our task is closer to the sociocultural use of the term. However, our study does not presuppose that the origin of the distinction in motion between males and females is of sociocultural or biological nature, as pointed out by Deaux [1993].

4.1.2 Emotion Recognition. We introduced the second block to explore and evaluate the accuracy of emotion portrayal by the actors. The effect of emotion on gender can only be justified if the emotions are correctly identified above chance level.

In this block, participants categorized the displayed emotion. They were asked "Which of the 5 listed emotions is the character expressing?" and selected an emotion by pressing the corresponding key on the keyboard, marked with A (Anger), F (Fear), H (Happy), S (Sad), and N (Neutral). We instructed them to use the Neutral condition only when they believed that the character did not express any emotion. They were also informed at the beginning of the experiment that one of the listed emotions would always be portrayed in each clip.

\subsection{Results}

Results were analyzed using repeated measures Analysis of variance (ANOVA) with within-subjects factors Actor Sex, Model, and Emotion, and between-groups factor Participant Sex on all of our data. 


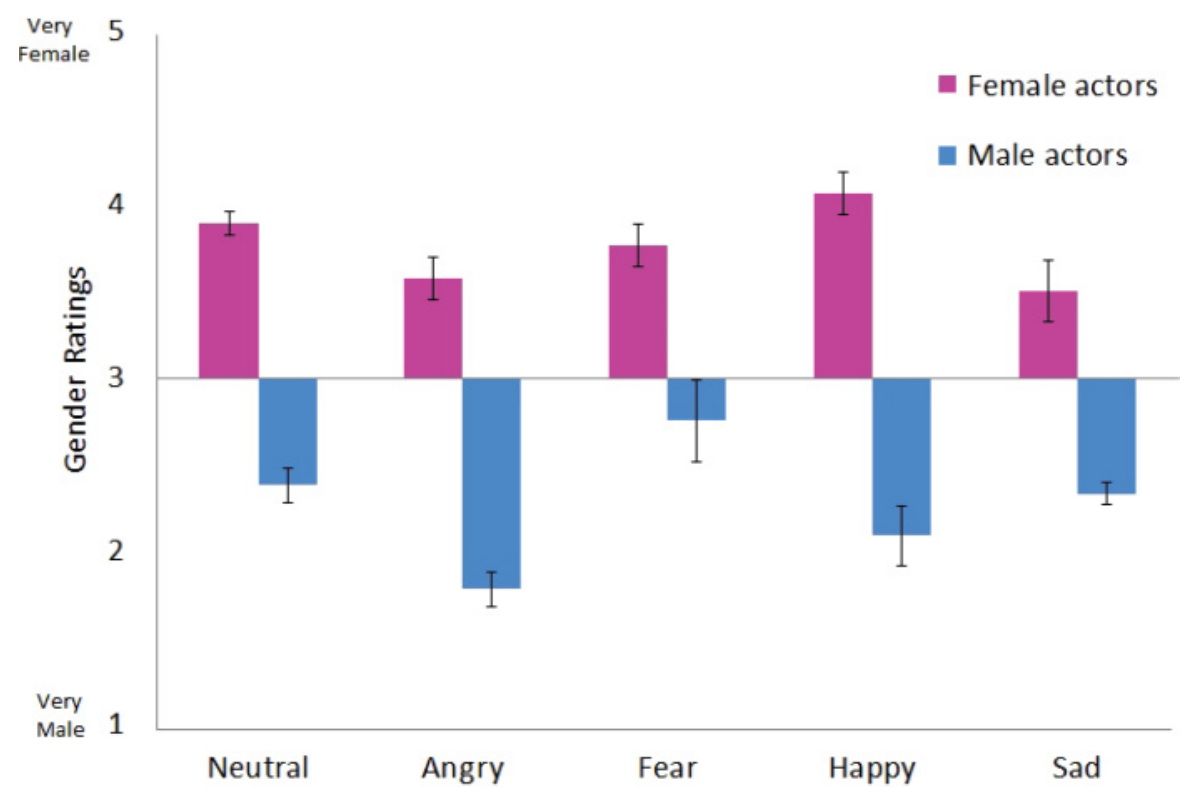

Fig. 2. Experiment 1: An interaction between Actor Sex and Emotion according to gender ratings for walking motions. Error bars show standard error of the means.

In all cases, we averaged over the data for the four actors for Actor Sex. For significant differences, estimates of effect size are reported using partial eta-squared. Where important interaction effects were found, we conducted Newman-Keuls tests for comparison of means to further explore the results. Degrees of freedom have been corrected using Greenhouse-Geisser estimates of sphericity, and Levene's test was used for testing homogeneity of variances. For clarity, we present the results grouped for each block (Gender Rating and Emotion Recognition) with the results relevant to our research in the form of questions and answers.

4.2.1 Gender Rating. We first investigated the results for gender ratings. The participants were able to distinguish between male (average: $2.27 \pm 0.08$ ) and female (average: $3.76 \pm 0.10$ ) motions overall $\left(F(1,22)=85.627, p \approx 0, \eta_{p}^{2}=0.796\right)$. This ability to rate the gender of the motion was unaffected by the character model used to present the motion. Overall, it is possible to recognize gender from walking motions, as previous studies have shown [Johansson 1973; Kozlowski and Cutting 1977].

Does emotion affect gender rating? Ratings for gender of the motion were affected by Emotion ( $F(2.7$, $58.5)=10.170, p \approx 0, \eta_{p}^{2}=0.316$, Greenhouse-Geisser correction $(\epsilon=0.67)$ ). Post hoc analysis showed that Angry (average: $2.68 \pm 0.07$ ) was considered significantly more male than Sad (average: $2.91 \pm 0.08$ ), Happy (average: $3.08 \pm 0.05$ ), Neutral (average: $3.13 \pm 0.06$ ), and Fear (average: $3.26 \pm$ $0.10)$, with $p<0.01$ in all cases. Sad was also found to be significantly more male than Fear $(p<0.002)$ and Neutral $(p<0.05)$. These results show that different emotional conditions affect gender ratings.

An interaction also occurred between Actor Sex and Emotion $\left(F(4,88)=17.434, p \approx 0, \eta_{p}^{2}=0.442\right)$ (see Figure 2), where post hoc analysis showed that all emotions portrayed by female actors were rated as significantly more female than their male counterparts ( $p<0.0003$ in all cases). For females, Sad and Angry ( $p<0.03$ ) were rated as the least female emotions, followed by Fear $(p<0.03$ in all cases). Neutral and Happy were rated as the most female emotions ( $p<0.03$ in all cases), where Happy was 
rated as more female than Neutral but the difference is not significant. For males, Fear was rated as the least male motion ( $p<0.0002$ in all cases), and Sad and Neutral were rated equally $(p<0.009$ in all cases) but less male than Happy ( $p<0.008$ in all cases). Angry was considered the most male of all motions ( $p<0.001$ in all cases). Angry was therefore perceived as the most male for male and the least female for female motions, which confirms the studies on the effect of gender stereotypes on production and perception of emotion [Fischer 1993] and previous findings on gender bias from body motion [Johnson et al. 2011]. Therefore, gender ratings for some emotional conditions show a possible influence of stereotypes on gender perception. This perception bias is further discussed in Section 6.2.

To determine whether participants could identify gender of the motion from different emotional conditions, we decided to compare the mean gender ratings for male and female motion with the average rating, which indicates the absence of visible gender cues. To test this, we conducted one-sample $t$-tests for each Emotion $\times$ Actor Sex combination (i.e., gender ratings significantly different from the constant 3). The ratings for male and female Angry, Neutral, Sad, and Happy were significantly different from the middle estimate of gender ( $p \approx 0$ for all). Male Fear was seen as less male but still significantly different from being rated as ambiguous $(t(15)=-2.6, p<0.02)$. We therefore conclude that emotion affects gender judgments of walking motions, but these changes never differ greatly from the actor's actual sex.

Does appearance of the model influence gender ratings? There was no main effect of Model on gender ratings, but an interaction with Model and Emotion was found $\left(F(4,88)=3.174, p=0.017, \eta_{p}^{2}=\right.$ 0.126), where Happy and Angry were seen as more male on the Woman model than on the Man model $(p<0.02$ for all). Previous research [Hess et al. 2004] showed that anger on a female face looks more intense than on a male face due to the contrast effect, as anger is perceived to be a male emotion. Our results might be showing this contrast effect as well, since Angry on the Woman model appeared more male. However, this effect was not found for other emotion and model combinations.

There was also an interaction between Participant Sex, Actor Sex, and Model $(F(1,22)=11.132, p=$ $0.003, \eta_{p}^{2}=0.336$ ). Female participants could identify male walks on the Woman model more easy than on the Man model $(p<0.008)$. A similar result was found for male participants, who found female walkers easier to identify on the Man model than on the Woman model $(p<0.02)$. These results might also be related to the contrast effect mentioned previously [Hess et al. 2004], as it appears to have been easier for participants to identify the gender of the actor of opposite sex displayed on an incongruent model. However, it remains uncertain as to why the sex of the observer matters in this interaction. We can conclude that the appearance of the model affects perception of gender. These results could also be highly related to the contrast effect; however, further experiments will be necessary to fully explore this relationship.

4.2.2 Emotion Recognition. To explore the role of emotions and to test if the emotions were correctly identified above chance level by the participants, we analyzed results for emotion recognition.

Were basic emotions correctly portrayed by walking motions? Overall, participants were very accurate at identifying emotions ( $70 \%$ accuracy on average), with $20 \%$ being the chance level. However, we found a main effect of Emotion $\left(F(2.4,51.6)=20.758, p \approx 0, \eta_{p}^{2}=0.485\right.$; Greenhouse-Geisser correction $(\epsilon=0.59))$, and post hoc analysis showed that Neutral and Sad were significantly more recognized than any other emotion ( $p<0.005$ in all cases). The next most recognized was Fear $(p<0.03$ in all cases), followed by Angry and Happy, which were the least recognized emotions ( $p<0.03$ in all cases). It is possible that the Sad emotion was easiest to recognize, due to the significant head cue (lowering of the head), which could only be present for this emotion. Neutral walk was highly recognized, but other emotions were more likely to be confused with Neutral as well (Table I shows the confusion matrix 
for Experiment 1), especially in the case of Happy and Angry. This result is similar to the study of Crane and Gross [2007], where they found sadness being the most and anger the least recognized of the tested emotions. Since we have not used facial motion for our stimuli, this effect could be attributed to the lack of facial information, which is further discussed in Section 6.2. Overall, our results show that basic emotions were accurately portrayed through walking motion.

Does sex of the actor affect the recognition of emotion? Since studies have found that females are better at portraying emotions [Brody and Hall 2000], we analyzed our results for possible differences in emotion recognition in relation to the actor's sex. We found a main effect of Actor Sex $(F(1,22)=$ 41.137, $p \approx 0, \eta_{p}^{2}=0.651$ ), which showed that participants were more accurate at emotion recognition when viewing female motion $(77.29 \% \pm 0.04)$ than male motion $(63.54 \% \pm 0.03)$. An interaction between Actor Sex and Emotion $\left(F(2.9,62.4)=27.398, p \approx 0, \eta_{p}^{2}=0.554\right.$; Greenhouse-Geisser correction $(\epsilon=0.71)$ ) showed that this was because the emotions Angry, Fear, and Happy were more easily recognized on the female actor ( $p<0.003$ in all cases; see Figure 6, blue series). Participants confused both male Angry and Happy walk with Neutral, and male Fear walk was often mistaken with Sad. The recognition rates and misclassifications for each emotion according to the actor's sex are seen in the confusion matrix (see Table I). This shows that emotions expressed by females through walking are easier to recognize than emotions expressed by males.

Does the sex of the virtual model change the perception of emotion? Appearance can also influence emotion perception [Hess et al. 2004], so we were interested in the differences in emotion recognition for the models we used. There was no main effect on the accuracy of emotion recognition, but we found a slight interaction with Emotion $\left(F(4,88)=2.478, p<0.049, \eta_{p}^{2}=0.101\right)$ where the Man model was slightly better for recognizing Fear (73\% on the Man model and 67\% on the Woman model) but was worse for recognizing Angry than the Woman model (58\% on the Man model and $64 \%$ on the Woman model; $p<0.05$ for all). Therefore, the appearance of the model has a slight influence on how specific emotions are perceived.

\section{EXPERIMENT 2}

In Experiment 1, we found that the underlying sex of the actor is recognized even if actors are expressing emotions that could bias the perceived gender. We hypothesize that this finding is due to the existence of strong motion cues in walking that make the perception of gender less prone to emotional bias. Therefore, in this experiment, we focus on another type of motion-conversation, which does not rely as heavily on the physiological differences between sexes as walking does. We studied the same interaction: how basic emotions affect gender perception of conversational motion and how the choice of model affects this interaction. As in Experiment 1, we also assessed the accuracy of the recognized emotions by asking the participants to report which emotions the characters were expressing.

\subsection{Experiment Design}

Similar to Experiment 1, participants rated the gender of the character based on their perception of motion in the first block. In the second block, they were asked to categorize the type of emotion the virtual character was expressing. In both blocks, participants were presented with the same set of randomized 240 video clips depicting virtual characters conveying different emotional sentences (Figure 3). In each block, half of the participants viewed the Man model stimuli first and the other half viewed the Woman model first. Trials lasted 3 seconds each, and participants were not presented with audio. In total, participants saw 240 animations in each block: 2 Models (Woman, Man) $\times 8$ Actors $(4 \mathrm{~F}, 4 \mathrm{M}) \times 5$ Emotions (Angry, Fear, Happy, Sad, and Neutral) $\times 3$ Sentences. Sixteen participants (8M, $8 \mathrm{~F}$; average age: $31 \pm 6.6$ years) took part in each block of this experiment. Fifteen participants 

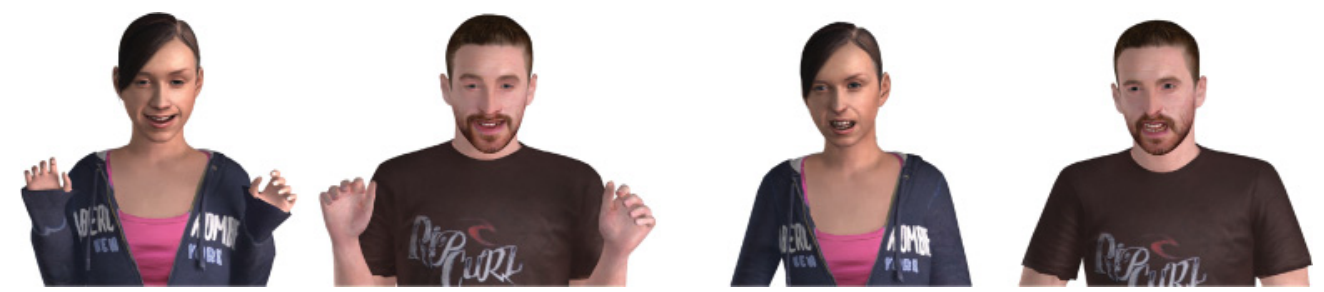

Fig. 3. Stimuli for Experiment 2. Left: Female motion applied to the Woman and Man models (Happy emotion). Right: Male motion applied to both models (Angry emotion).

completed both blocks (7M, 8F), and two additional participants completed just one block each. They were recruited mainly from the university, had normal or corrected-to-normal vision, and were all naïve to the purpose of the experiment. University ethical approval was granted for the experiment, participants read and signed the informed consent, and they received a $€ 5$ book voucher to compensate for their time.

\subsection{Results}

As in Experiment 1, results for both the Gender Rating and Emotion Recognition tasks were analyzed using repeated measures ANOVA with within-subjects factors Actor Sex, Model, and Emotion, and between-groups factor Participant Sex on all of our data. In all cases, we averaged over the data for the four actors for Actor Sex and over the three sentences. For significant differences, estimates of effect size are reported using partial eta-squared. Degrees of freedom have been corrected using GreenhouseGeisser estimates of sphericity, and Levene's test was used for testing homogeneity of variances. Where interaction effects were found, we conducted Newman-Keuls tests for comparison of means to further explore the results. No effect of Participant Sex was observed for any tests conducted, so this is not discussed further.

5.2.1 Gender Rating. As in Experiment 1, we found that participants were able to distinguish between male (average: $2.53 \pm 0.03$ ) and female (average: $3.49 \pm 0.05)$ motions overall $(F(1,14)=$ $244.260, p \approx 0, \eta_{p}^{2}=0.945$ ). This implies that it is possible to recognize gender from conversational motions as well, which confirms the findings of previous studies [Hill et al. 2003].

Does emotion affect gender rating? We were mainly interested in the effect of emotion on gender ratings. We found that the ability to rate the gender of a motion was affected by Emotion $(F(1.6,22.4)=$ 19.426, $p \approx 0, \eta_{p}^{2}=0.581$; Greenhouse-Geisser correction $(\epsilon=0.40)$ ). In general, Angry (average: $2.47 \pm 0.11$ ) was considered significantly more male than Neutral (average: $3.03 \pm 0.08$ ), Fear (average: $3.09 \pm 0.05$ ), Happy (average: $3.24 \pm 0.05$ ), or Sad (average: $3.20 \pm 0.07$ ). There were no significant differences in gender ratings for other emotions. As in Experiment 1, we found that emotions affect gender ratings.

An interaction occurred between Actor Sex and Emotion $\left(F(4,56)=53.197, p \approx 0, \eta_{p}^{2}=0.791\right)$ (Figure 4) as well, where post hoc analysis showed that all emotions expressed by female actors were rated as significantly more female than their male counterparts ( $p<0.0003$ in all cases). For females, Angry was rated as significantly less female than all other emotions ( $p<0.0003$ in all cases). Neutral was rated as more female ( $p<0.0008$ in all cases), followed by Fear and Sad, which were rated equally ( $p<0.0008$ in all cases). Happy was rated significantly more female than all other emotions ( $p<0.0002$ in all cases). For males, Sad and Neutral were rated equally as the least male of all emotions ( $p<0.009$ in all cases). Fear was considered more male ( $p<0.009$ in all cases), followed 


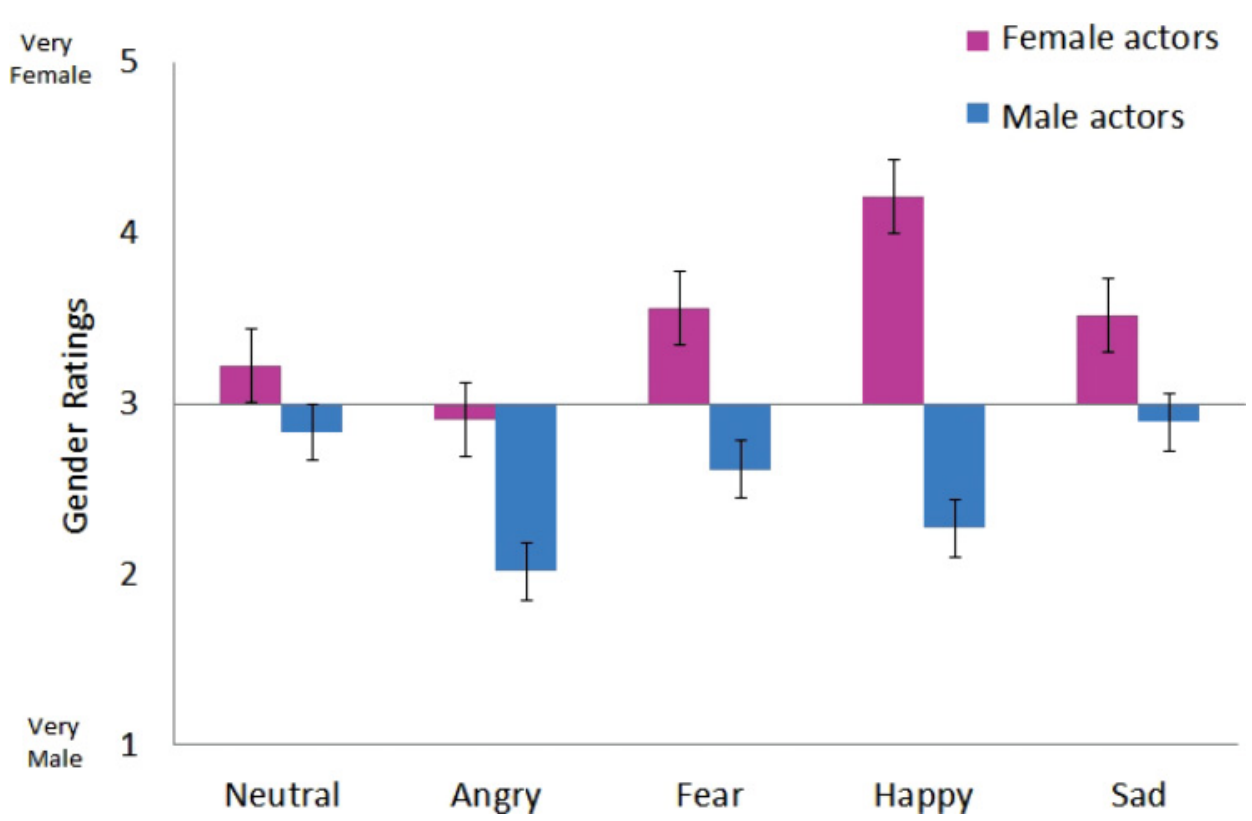

Fig. 4. Experiment 2: An interaction between Actor Sex and Emotion on gender ratings for conversational motions. Error bars show standard error of the means.

by Happy ( $p<0.003$ in all cases), and finally Angry was rated as the most male of all male emotions ( $p<0.003$ in all cases). Therefore, it might be more difficult to identify gender when a female actor is expressing anger, and for male expressions of sadness and neutral sentences. Happiness, however, appears to facilitate the recognition of gender for both males and females. The possible influence of stereotypes on gender perception is further discussed in Section 6.2.

As in Experiment 1, we checked whether participants could identify gender by conducting onesample $t$-tests for each Emotion $\times$ Actor Sex combination (i.e., gender ratings significantly different from the constant 3). We found that for Angry, participants could not determine the gender of the female motions, but they could do so for male motions $(t(15)=-9.63, p \approx 0)$. For Neutral and Sad, male motions did not convey gender information, but female motions did (Sad: $t(15)=5.93, p \approx 0$; Neutral: $t(15)=3.14, p<0.007)$. For Happy and Fear, male motions were recognized as male (Happy: $t(15)=-10.724, p \approx 0$; Fear: $t(15)=-6.154, p \approx 0$ ) and female motions as female (Happy: $t(15)=$ 13.809, $p \approx 0$; Fear: $t(15)=7.927, p \approx 0$ ). Therefore, some gender-specific emotions (female anger, male neutral, and male sadness) prevented an accurate recognition of gender. However, female actors were generally not rated as male, and male actors were not perceived to be female. We conclude that emotions affect gender judgments of conversational motions but do not cause misperceptions of gender.

Does appearance of the model influence gender ratings? Finally, the model used to present the emotions did not affect ratings (no main or interaction effects). These results imply that appearance does not affect gender perception of conversing characters.

5.2.2 Emotion Recognition. As in Experiment 1, we included the emotion recognition task and validated that our sets of conversational motions are correctly identified above chance level.

Were basic emotions correctly portrayed by conversing characters? We found that overall, participants were very accurate in identifying the acted emotions (68\% accuracy on average), with $20 \%$ being 
Table I. Confusion Matrix: Emotion Recognition for Walking Motions (Experiment 1) and Conversational Motions (Experiment 2) Averaged over Model

\begin{tabular}{|c|c|c|c|c|c|c|c|c|c|c|c|}
\hline & & \multicolumn{5}{|c|}{ EMOTION RECOGNITION (Experiment 1) } & \multicolumn{5}{|c|}{ EMOTION RECOGNITION (Experiment 2) } \\
\hline & & Neutral (\%) & Angry (\%) & Fear (\%) & Happy (\%) & Sad (\%) & Neutral (\%) & Angry (\%) & Fear $(\%)$ & Happy (\%) & Sad (\%) \\
\hline \multirow{5}{*}{$\begin{array}{c}\text { FEMALE } \\
\text { actor }\end{array}$} & Neutral & 82.8 & 1.6 & 4.7 & 8.3 & 2.6 & 59.1 & 5.2 & 3.4 & 3.2 & 29.2 \\
\hline & Angry & 10.9 & 73.4 & 5.2 & 9.4 & 1.0 & 0.5 & 95.6 & 2.1 & 0.0 & 1.9 \\
\hline & Fear & 4.2 & 1.0 & 92.7 & 1.0 & 1.0 & 1.6 & 6.5 & 79.2 & 1.3 & 11.5 \\
\hline & Hарру & 21.9 & 3.6 & 9.9 & 63.5 & 1.0 & 1.8 & 5.5 & 0.8 & 89.1 & 2.9 \\
\hline & Sad & 17.2 & 2.1 & 4.7 & 2.1 & 74.0 & 11.7 & 4.5 & 8.4 & 1.1 & 74.5 \\
\hline \multirow{5}{*}{$\begin{array}{l}\text { MALE } \\
\text { actor }\end{array}$} & Neutral & 86.5 & 3.1 & 1.0 & 6.3 & 3.1 & 46.4 & 9.1 & 2.1 & 9.4 & 33.1 \\
\hline & Angry & 36.5 & 48.4 & 5.2 & 2.6 & 7.3 & 0.3 & 96.1 & 2.9 & 0.3 & 0.5 \\
\hline & Fear & 6.8 & 6.8 & 47.9 & 1.6 & 37.0 & 8.9 & 24.2 & 52.4 & 3.4 & 11.3 \\
\hline & Hарру & 26.6 & 24.0 & 1.0 & 44.8 & 3.6 & 3.2 & 9.1 & 0.3 & 86.8 & 0.8 \\
\hline & Sad & 7.8 & 0.0 & 1.0 & 10 & 90.1 & 24.0 & 8.9 & 9.2 & 4.2 & 53.9 \\
\hline
\end{tabular}

the probability of recognition due to chance. We also found a main effect of Emotion $(F(1.5,20.8)=$ 35.389, $p \approx 0, \eta_{p}^{2}=0.716$; Greenhouse-Geisser correction $\left.(\epsilon=0.37)\right)$, and post hoc analysis showed that Angry and Happy were significantly more recognized than any other emotion $(p<0.0002$ in all cases). Sad and Fear were rated equally accurately, and Neutral was the least recognized emotion ( $p<0.009$ in all cases). As in Experiment 1, basic emotions are recognized from conversational motions but with different accuracy.

Does the sex of the actor affect the recognition of emotion? We found a main effect of Actor Sex $\left(F(1,14)=179.90, p \approx 0, \eta_{p}^{2}=0.928\right)$, which showed that participants were more accurate at emotion recognition when viewing female motions $(79.48 \% \pm 1.36)$ than male motions $(67.09 \% \pm 1.48)$. An interaction between Actor Sex and Emotion $\left(F(4,65)=28.964, p \approx 0, \eta_{p}^{2}=0.674\right)$ showed that this was because all emotions except Angry and Happy were more easily recognized on the female actor $(p<0.04$ in all cases; see Figure 6, red series). The confusion matrix shows where misclassifications occurred (Table I). From these results, we can conclude that females are better at portraying conversational emotions than males. Specifically, male sadness, fear, and neutral portrayals are less recognizable from conversation. Anger is stereotypically a male emotion, so it is not surprising that it was correctly portrayed by males. Even though happiness is more attributed to females, happiness might be easier to portray with facial motion by both males and females. This explanation is supported by the study of Ennis et al. [2013], where they demonstrated that facial motion is important in disambiguating happy and angry emotions from body motions.

Does appearance influence emotion recognition? Model was found to have an effect on accuracy $\left(F(1,14)=9.177, p<0.009, \eta_{p}^{2}=0.395\right)$, with motion recognition being more accurate on the Man model $(74.74 \% \pm 1.45)$ than on the Woman model $(71.83 \% \pm 1.41)$. We also found an interaction between Emotion and Model $\left(F(2.0,28.0)=10.880, p \approx 0, \eta_{p}^{2}=0.437\right.$; Greenhouse-Geisser correction $(\epsilon=0.50)$ ), which was because Fear and Sad were detected more easily on the Man than on the Woman ( $p<0.0002$ for both). Therefore, virtual model has an influence on how specific emotions are perceived.

\section{CROSS-EXPERIMENTAL ANALYSIS}

We conclude our analysis of results by comparing the results of Experiments 1 and 2. This was done to evaluate if the type of motion (walking and conversation) had any effect on how emotions affect gender perception. We hypothesized that conversational motion would be more prone to gender bias because 
it does not contain as many gender cues as walking motion, so the gender would be more difficult to determine and emotions would have a stronger effect on gender ratings. We also compared emotion recognition accuracy for both motion types.

\subsection{Results}

To understand how the two different types of motions-walking and conversing-affect the gender and emotion interaction, we further analyzed the results of Experiments 1 and 2 in relation to each other. We first conducted repeated measures ANOVA with within-subjects factors Actor Sex, Emotion, and Model, and between-groups factors Motion Type and Participant Sex on all of our data from both experiments. In Experiment 2, we used three different sentences to portray each emotion for variety, as our actors portrayed conversational emotions differently across sentences. In Experiment 1, we used one example of a walk per emotion, as our actors were consistent in their portrayal of emotion across walks. Therefore, participants in Experiment 2 saw three times more stimuli than in Experiment 1. However, since the order of stimuli was randomized and the task was not one that could be learned as the experiment progressed, we conducted cross-experimental analysis by using a mixed-model ANOVA. To conduct ANOVA, we averaged ratings over the three sentences from Experiment 1 to give us an average rating of emotion. As before, we tested for assumptions of ANOVA, using Greenhouse-Geisser correction for sphericity and Levene's test for homogeneity of variance, and used transformations on the raw data where assumptions were not met. If this was not successful, we used nonparametric tests (Wilcoxon signed-rank test for repeated measures and Mann-Whitney U test for comparisons of independent samples). Where assumptions were met, the Newman-Keuls test was used for comparison of means to further explore interaction effects.

6.1.1 Gender Ratings. Main effects were investigated to explore how the effects of emotion, sex of the actor, and model could influence the results regardless of the Motion Type, for which no main effect was found. A main effect was found for Actor $\operatorname{Sex}\left(F(1,36)=120.80, p \approx 0, \eta_{p}^{2}=0.770\right)$, which shows that gender was rated according to the underlying sex. This result was expected because both previous results from Experiments 1 and 2 showed this effect. The other main effect was found for Emotion $\left(F(3.1,110.3)=24.273, p \approx 0, \eta_{p}^{2}=0.402\right.$; Greenhouse-Geisser correction $\left.(\epsilon=0.76)\right)$. The post hoc conducted for the second effect indicates that overall, Angry was rated as significantly more male than any other emotion, with the average rating of $2.60 \pm 0.06$. Two interactions were found as well: one between Actor Sex and Emotion $\left(F(4,144)=41.099, p \approx 0, \eta_{p}^{2}=0.533\right)$, where similar patterns of gender ratings emerged as in Experiments 1 and 2, and one between Actor Sex, Model, and Participant Sex $\left(F(1,36)=5.312, p=0.027, \eta_{p}^{2}=0.128\right)$ as in Experiment 1.

Is the underlying sex of the actor easier to detect on walking or conversational motions? We assumed that the actor's sex would be more apparent from walking than conversational motions, since identifiable sex cues exist in walking motions that make sex identification easier and less prone to gender bias. A significant interaction between Motion Type and Actor Sex occurred $(F(1,36)=4.527, p=$ $0.040, \eta_{p}^{2}=0.111$ ), where males were rated more male and females more female on walking motions than on conversational motions. This result shows that actor's sex is indeed more apparent on walking than on conversational motions.

Are conversational motions more prone to the gender bias that exists around emotions than walking motions? We found an interaction between Emotion and Motion Type $(F(3.1,110.3)=5.341, p=$ $0.002, \eta_{p}^{2}=0.129$; Greenhouse-Geisser correction $(\epsilon=0.76)$, where post hoc analysis revealed that Angry was rated significantly more male for conversers than walkers, and an Actor Sex, Motion Type, and Emotion interaction $\left(F(4,144)=13.330, p \approx 0, \eta_{p}^{2}=0.270\right)$, where post hoc analysis showed 

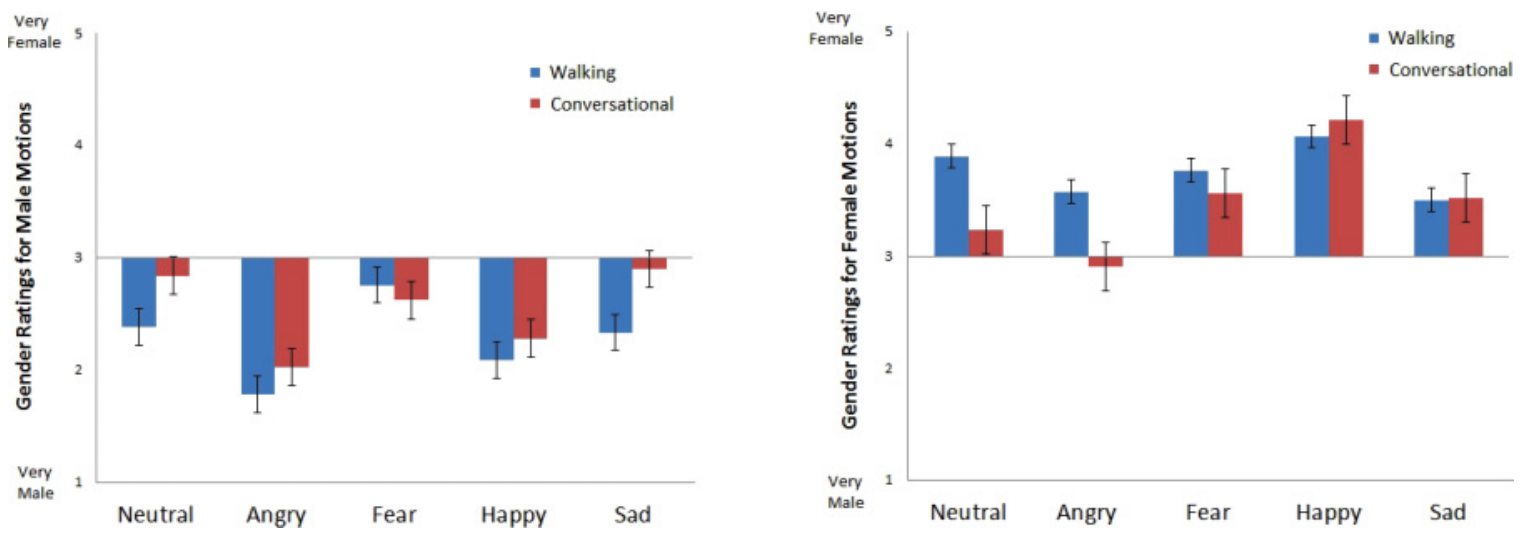

Fig. 5. Comparison of interactions between Emotion and Motion Types (walking and conversation) on gender ratings for male motions (left graph) and female (right graph) motions. Error bars show standard errors of mean gender ratings.

that gender was better recognized for walking motions in the case of Angry, Sad, and Neutral male motions ( $p<0.02$ for all) and Neutral and Angry female motions ( $p<0.0002$ for all). However, no differences were found between Happy and Fear. Gender biases could be observed in Sad (male) and Angry (female) conditions, where the gender of the conversers was perceived as more ambiguous (Figure 5) than in the walking condition. We also expected that participants would be better at recognizing gender from the Neutral walking condition than in conversation, because of differences in gender cues between conversations and walking, and our results confirm this expectation. We can therefore conclude that where differences in gender perception between the two types of motion were found, they showed a greater influence of emotion on gender stereotypes.

Does appearance of the model influence gender ratings for both types of motion? As in previous experiments, we investigated the effect of model on gender ratings. We found only an interaction between Participant Sex, Actor Sex, Model, and Motion Type $\left(F(4,152)=2.556, p=0.041, \eta_{p}^{2}=0.169\right)$, again, showing a slight contrast effect when female participants rated male motion on a female actor as more male and male participants rated female motion on a male actor as more male. However, this effect was prominent only for walking motions ( $p<0.03$ for both). Thus, appearance of the model has a slight effect, which depends on the type of motion and sex of the observer.

6.1.2 Emotion Recognition. For this set of data, the assumptions for ANOVA were not met and transformations on our data were not successful; therefore, we used the nonparametric Mann-Whitney $\mathrm{U}$ test for independent samples to compare variables of both groups and report significant differences. First, we were interested to determine if there were significant differences on recognition of emotion according to Participant Sex, but we found no differences. We therefore continued to analyze recognition accuracy for male and female actors separately, averaged over models, to explore the differences between recognition accuracy of the two motion types. Finally, we used the Wilcoxon signed-rank test for repeated measures to compare differences in recognition accuracy according to Model.

Are emotions, expressed through different types of motions, recognized with the same accuracy? There are significant differences for all emotions between the two types of motion, except for Sad expressed by female actors and Fear portrayed by male actors (Figure 6). Walking was better for the recognition of Neutral (Male actors: $U=34.5, p \approx 0$; Female actors: $U=67.5, p=0.001$ ), Fear expressed by females $(U=50.5, p \approx 0)$, and Sad expressed by male actors $(U=16.0, p \approx 0)$. Angry and Happy 

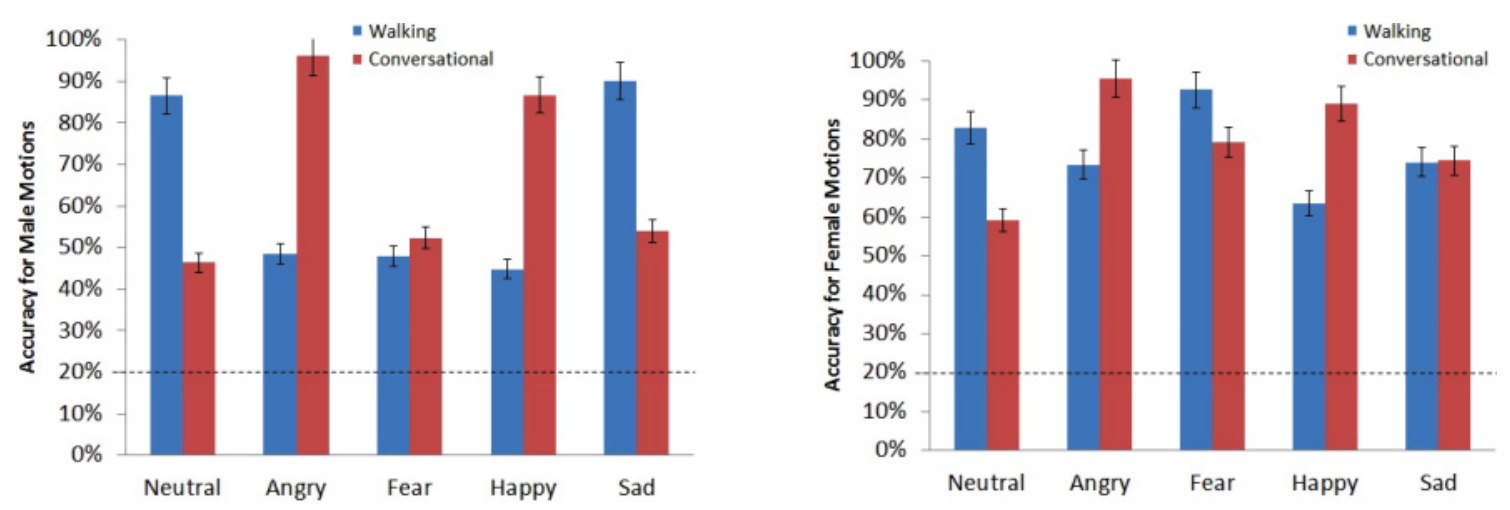

Fig. 6. Comparison of means between different types of motion (walking and conversation) for male actors (left graph) and female actors (right graph) on emotion recognition. The dashed line represents chance level of recognition (20\% accuracy); error bars show standard error of the means.

emotions were better recognized from conversation (Male Angry: $U=0.0, p \approx 0$, and Male Happy: $U=7.5, p \approx 0$; Female Angry: $U=86.0, p=0.003$, and Female Happy: $U=44.0, p \approx 0)$. Thus, overall, neutral motions are easier to recognize on walking, whereas anger and happiness are more recognized on conversational motions.

Does appearance of the model influence emotion recognition for both types of motion? Significant differences between Model were found according to Motion Type, Emotion, and Actor Sex. The Man model improved the recognition for conversational motions in the cases of Sad (for males: $Z=2.020, p=$ 0.042 ; for females: $Z=2.301, p=0.021)$ and female Fear $(Z=2.481, p=0.013)$. For walking motions, the Man model improved the recognition for male Neutral motion $(Z=2.030, p=0.042)$. The Man model also improved accuracy for male Fear for both types of motions (conversation: $Z=2.950, p=$ 0.003; walking: $Z=2.310, p=0.021$ ). The Woman model improved recognition only for female Angry conversational motion $(Z=2.401, p=0.016)$. These results might show a slight preference of the Man model when it comes to accuracy, especially for conversational motions and emotions that are stereotypically not attributed to males (Fear, Sad). Similarly, the Woman model improved the recognition of Angry conversation, an emotion that typically is attributed to males. Further tests would have to be conducted to understand why the model would have this effect for these specific conditions. Our results therefore indicate that some models are better for the recognition of emotions according to a particular motion type than others.

\subsection{Discussion}

The results of the cross analysis show that for both walking and conversational motions, gender is generally rated according to the underlying actor's sex. In addition, both motions presented some variations in gender perception when emotional conditions were introduced. These variations, as we predicted, were mostly in favor of the hypothesis that certain emotions are attributed more to one gender than the other. This effect was most prominent for anger, an emotion commonly attributed to males [Brody and Hall 2000] and was perceived as more male when expressed by male actors and perceived less female when expressed by female actors. Similarly, sadness and fear, which are commonly attributed to females, caused male motions to appear less male overall. The only emotion that did not follow our hypothesis was happiness, which is an emotion commonly attributed to females. Unexpectedly, happy motions carried gender information even for males. Therefore, it is possible that there are 
specific ways in which males and females express this emotion that could explain why stereotypes did not have a stronger effect. From our motion capture session, it was possible to notice that female actors were expressing happiness more freely, whereas male actors were more reserved. Given the fact that happiness was properly recognized overall, we believe that this difference in the way in which happiness was portrayed by the actors might have made gender easier to identify.

In our study, we were particularly interested in the differences between walking and conversational motions. We argued that motions containing fewer gender cues would be more affected by gender stereotypes that exist around certain emotions. First, we noticed a difference in gender judgments for neutral conditions between both types of motion, which confirmed our belief that gender was less apparent in conversational than walking motions. We assumed that this was due to the presence of physiological gender cues in walking as opposed to less obvious or absent gender cues in conversation. Furthermore, in certain emotional conditions, gender bias appeared to be more prominent for conversing characters: male sadness was rated as significantly less male in the conversation than in the walking condition, and female anger was perceived to be less female in conversation as opposed to walking. However, the gender was not perceived as coming from the opposite sex, which means there might still be some gender information present in the conversational motion that would prevent stereotypes from completely influencing the judgment. This result is different from the result of Johnson et al. [2011], in which they found that angry throwing motions resulted in the perception of the actor's sex being perceived as male, even when female actors were portraying it. Based on our assumptions, throwing motion may not contain as many gender cues as conversations, and therefore gender judgments in their study could have relied mostly on the portrayed emotion. In the case of happiness and fear, we expected male actors to appear less male in the conversational motion as opposed to the walking motion. However, we found no difference in the effect of gender bias for these two motion types.

The actual appearance of the model also had a small effect on the perception of gender. Male participants perceived female walking as more female on the Man model, and female participants perceived male walking more male on the Woman model. These results show a slight contrast effect, as proposed by Hess et al. [2004]. Furthermore, the role of participant sex in assessing the gender of the opposite sex could be explained with mating strategies proposed by evolutionary psychology (for an overview, see Hugill et al. [2010]). Since reproduction and selectivity of partners is an important task, humans have a high sensitivity for assessing nonverbal cues from the people of the sex to which they are attracted. It could be that male participants in our study were more sensitive toward female motion, which became even more apparent on male models, and female participants spotted male motion on a female model more easily as well. However, the exact role of the virtual model, participant sex, and motion type should be investigated with further research, using more examples and systematic changes of the stimuli.

Confusion with other emotions gave us information on how well the emotions were perceived by participants. All emotions were recognized above chance, confirming that the variation in our gender judgment tasks were due to the target emotions. Even though all emotions were recognized above chance, and as known from the literature [Brody and Hall 2000], females are better at portraying emotions, which might make the emotion categorization task easier. Even though emotions expressed by male actors were more often confused with each other, they were considerably well recognized in our study.

We also found that emotions were more difficult to recognize on one but not the other type of motion. Male anger and happiness were more difficult to recognize on walking motions, whereas they were the most recognized emotions in conversation. This could mean that some types of motions are better for emotion portrayal or that there are certain areas of the body that are more important for the expression of certain emotions-for example, happiness is more difficult to identify if no facial information is 
present. This is not an unusual result, as previous studies have found different recognition rates for emotion from body, facial, and combined expressions [Clavel et al. 2009; Aviezer et al. 2012; Ennis et al. 2013]. Particularly interesting is the study of Aviezer et al. [2012], in which highly intense emotional expressions taken from real-life situations were correctly identified from body motion but not from isolated facial expressions. Therefore, it appears that intensity of emotion plays a part in recognition from certain parts of body.

Male fear appeared to be the only emotion that was difficult to recognize in both types of motions. This could mean that male actors were not as successful at portraying fear as female actors. It could be an indication of a cultural effect on the production of the emotion. For example, males are not expected to portray fear, and therefore their portrayal of this emotion was harder to categorize by the participants. Furthermore, as mentioned earlier, gender ratings showed more difficulties in perceiving the underlying gender when male actors were portraying fear. Both results could point to the cultural effect on the production and perception of emotion.

Even though we cannot explain how appearance affected gender ratings, we found that the choice of model could improve emotion recognition. Some emotions (mainly fear and sadness) were easier to recognize on the Man model. Again, further studies, using more models, might reveal what contributed to this result.

\section{GENERAL DISCUSSION}

In this article, we introduced some new features to the understanding of how certain basic emotions can influence the perception of gender of virtual characters. We proposed that (1) stereotypes for emotion will bias gender perception, (2) this effect will be stronger for motions that do not exhibit obvious physiological cues, and (3) there would be an effect of virtual model on gender perception. Our results generally confirmed all three hypotheses. The way in which we perceive gender from motion does depend on whether specific emotion is expressed, how this emotion is expressed, and who expresses it. Some emotions are attributed more to one gender than the other and therefore can change our perception of gender, especially if the type of motion itself does not reveal the underlying gender sufficiently. In our case, anger and male sadness were the emotions that biased gender perception the most, especially when expressed through conversing rather than walking characters. Furthermore, the appearance of the model can also affect the perception of gender, particularly by making the underlying sex of the actor more apparent when it is mismatched with the sex of the virtual model.

However, in our study, emotions did not invert the perception of gender. Therefore, it is possible that attribution of emotion to a specific gender does not influence gender perception to a great extent when there are sufficient gender cues present in the motion already. A good rule to follow when creating virtual humans using natural human motion would thus be to match the actor's sex with that of the virtual character. But in cases or types of motions where gender is not apparent, some emotions can influence the perception of the actor's actual sex. For example, if we create a male virtual character, angry motion would make him look more male, whereas fearful and sad motion would make him appear less male.

Our result is also arguable from the point that we only found this effect for anger and male sadness but not other emotions. This could be because social stereotypes do not influence perception of emotion, but its production as well, as already suggested. For example, showing fear is seen as a sign of weakness; therefore, males would be more reserved while expressing it. This reservation in expression, and not the emotion itself, might make gender easier to identify by the observers. This would not show the effect of biased gender perception, but the very portrayal of emotion by an actor would be under the cultural influence on the production of the emotion. We believe that some emotion recognition rates in our experiment might have been lower because they were portrayed with more reservation as well. 
This possibility also prevented us to do gender judgment analysis only on best sets of recognized emotions, as that would artificially skew the data. A similar systematical bias on emotion recognition is presented in the paper of Johnson et al. [2011], where neutral, happy, and sad throwing motions were often confused with each other but angry was not. Overall, emotions in our experiment were recognized above chance, so we believe that the gender analysis per emotion was conducted as appropriately as possible given the intrinsic complexity of studying emotion and gender simultaneously.

Since we found a slight effect of the virtual character's appearance on the perception of gender, using the appropriate sex of the actor to match the appearance of the character can avoid any confusion. However, certain social subgroups, such as transsexuals, are shown to exhibit motion specifics of the sex with whom they identify themselves. It would be interesting to capture motions from these subgroups and compare them with the motions of people who exhibit their biological sex, applied to characters of the opposite sex. This could give us an idea if gender-specific motion is learned through identification with a specific sex group or if it relies on biological predispositions of the person. We also proposed that perceivers might have a higher sensitivity for the gender to which they are attracted [Hugill et al. 2010]. Hugill et al. [2010] It would thus be appropriate to explore certain characteristics, such as sexual orientation of the perceiver, in future work.

We are also aware of some limitations of our study. Due to technical difficulties, we could not capture finger or eye motion for the conversing characters, which may improve the believability of the motion, as the missing data can be detected from the observer [Jörg et al. 2010; Hodgins et al. 2010]. Due to the limitations of our motion capture system, we were also unable to record facial information for the walking motions, which might have improved emotional recognition and affected gender ratings. We are aware that in the cross analysis, we compared stimuli that are slightly different and that statistical differences could be due either to the lack of facial motion or differences in motion types. However, given the similar trends in the results of the cross analysis, such analysis already provides us insights about how emotions differ between different types of motions. Exploring if the complex additional facial features in walking animations affect these results would also be interesting in future experiments. Another limitation is that we only used two models to display the motion. More models would increase the reliability of the results and would add to the understanding of how appearance affects gender perception. Particularly, adding a genderless model or a point-light display (e.g., see studies of Wellerdiek et al. [2013] for the effect of model on motion perception, McDonnell et al. [2009] for sex judgments, and Atkinson et al. [2004] for emotion recognition) to the experimental stimuli could provide more information about the influence of appearance on the perceived motion. Furthermore, a gender rating study was used in our experiment design, where we measured judgments of gender, which could differ from actual perception of gender. Other measures (e.g., eye tracking) could give additional information about how we perceive gender of virtual humans. However, we are confident that the conducted research furthered our understanding about the effect of emotion on gender perception.

Future work will be oriented to overcoming the limitations of this study and expanding it to different types of motions, models, and situations. For example, we are interested in how emotions would change the perception of gender of characters in crowd simulations. It would be interesting to perform motion pattern analysis to see if male and female actors use fundamentally different motions to express different emotions. We would also like to explore other possible areas of social cognition apart from gender stereotypes to test if they can be associated with virtual humans.

The mentioned outcomes of our research can aid designers in improving believability of the characters and offer some insights into perception of natural motion, which is useful for anyone using motion capture systems for character animation. The outcomes of this study are also important to psychology and social studies. 


\section{REFERENCES}

Anthony P. Atkinson, Winand H. Dittrich, Andrew J. Gemmell, and Andrew W. Young. 2004. Emotion perception from dynamic and static body expressions in point-light and full-light displays. Perception 33, 6, 717-746.

Hillel Aviezer, Yaacov Trope, and Alexander Todorov. 2012. Body cues, not facial expressions, discriminate between intense positive and negative emotions. Science 338, 6111, 1225-1229.

John N. Bassili. 1978. Facial motion in the perception of faces and of emotional expression. Journal of Experimental PsychologyHuman Perception and Performance 4, 373-379.

Alberto Battocchi, Fabio Pianesi, and Dina Goren-Bar. 2005. A first evaluation study of a database of kinetic facial expressions (DaFEx). In Proceedings of the 7th International Conference on Multimodal Interfaces (ICMI'05). 214-221.

Boaz M. Ben-David, Pascal H. H. M. van Lieshout, and Talia Leszcz. 2011. A resource of validated affective and neutral sentences to assess identification of emotion in spoken language after a brain injury. Brain Injury 25, 2, 206-220.

Marilynn B. Brewer. 1988. A Dual Process Model of Impression Formation. Lawrence Erlbaum, Hillsdale, NJ.

Leslie R. Brody and Judith A. Hall. 2000. Gender, emotion, and expression. In Handbook of Emotions, M. Lewis and J. HavilandJones (Eds.). Guilford, 338-349.

Thierry Chaminade, Jessica Hodgins, and Mitsuo Kawato. 2007. Anthropomorphism influences perception of computer-animated characters' actions. Social Cognitive and Affective Neuroscience 2, 3, 206-216.

Céline Clavel, Justine Plessier, Jean-Claude Martin, Laurent Ach, and Benoit Morel. 2009. Combining facial and postural expressions of emotions in a virtual character. In Proceedings of the 9th International Conference on Intelligent Virtual Agents (IVA'09). 287-300.

Elizabeth Crane and Melissa Gross. 2007. Motion capture and emotion: Affect detection in whole body movement. In Affective Computing and Intelligent Interaction. Lecture Notes in Computer Science, Vol. 4738. Springer, 95-101.

James E. Cutting. 1978. Generation of synthetic male and female walkers through manipulation of a biomechanical invariant. Perception 7, 4, 393-405.

Marco De Meijer. 1989. The contribution of general features of body movement to the attribution of emotions. Journal of Nonverbal Behavior 13, 4, 247-268.

Kay Deaux. 1993. Commentary: Sorry, wrong number: A reply to gentile's call. Psychological Science 4, 2, $125-126$.

Paul Ekman. 1992. An argument for basic emotions. Cognition and Emotion 6, 3-4, 169-200.

Cathy Ennis, Ludovic Hoyet, Arjan Egges, and Rachel McDonnell. 2013. Emotion capture: Emotionally expressive characters for games. In Proceedings of Motion on Games (MIG'13). ACM, New York, NY, 53-60.

Agneta H. Fischer. 1993. Sex differences in emotionality: Fact or stereotype? Feminism and Psychology 3, 3, 303-318.

Agneta H. Fischer, Patricia M. Rodriguez Mosquera, Annelies E. M. Van Vianen, and Antony S. R. Manstead. 2004. Gender and culture differences in emotion. Emotion 4, 1, 87-94.

Ursula Hess, Reginald B. Adams, and Robert E. Kleck. 2004. Facial appearance, gender, and emotion expression. Emotion 4, 4, 378-388.

Harold Hill, Yuri Jinno, and Alan Johnston. 2003. Comparing solid-body with point-light animations. Perception 32, 5, 561-566.

Harold Hill and Alan Johnston. 2001. Categorizing sex and identity from the biological motion of faces. Current Biology 11, 11, 880-885.

Jessica Hodgins, Sophie Jörg, Carol O’Sullivan, Sang Il Park, and Moshe Mahler. 2010. The saliency of anomalies in animated human characters. ACM Transactions on Applied Perception 7, 4, 22:1-22:14.

Nadine Hugill, Bernhard Fink, and Nick Neave. 2010. The role of human body movements in mate selection. Evolutionary Psychology 8, 1, 66-89.

Gunnar Johansson. 1973. Visual perception of biological motion and a model for its analysis. Perception and Psychophysics 14, 2, 201-211.

Kerri L. Johnson, Lawrie S. McKay, and Frank E. Pollick. 2011. He throws like a girl (but only when he's sad): Emotion affects sex-decoding of biological motion displays. Cognition 119, 2, 265-280.

Kerri L. Johnson and Louis G. Tassinary. 2005. Perceiving sex directly and indirectly meaning in motion and morphology. Psychological Science 16, 11, 890-897.

Sophie Jörg, Jessica Hodgins, and Carol O'Sullivan. 2010. The perception of finger motions. In Proceedings of the 7th Symposium on Applied Perception in Graphics and Visualization. 129-133.

Lynn T. Kozlowski and James E. Cutting. 1977. Recognizing the sex of a walker from a dynamic point-light display. Perception and Psychophysics 21, 6, 575-580.

Rachel McDonnell, Sophie Jörg, Jessica K. Hodgins, Fiona Newell, and Carol O’Sullivan. 2009. Evaluating the effect of motion and body shape on the perceived sex of virtual characters. ACM Transactions on Applied Perception 5, 4, 20:1-20:14. 
Edward R. Morrison, Lisa Gralewski, Neill Campbell, and Ian S. Penton-Voak. 2007. Facial movement varies by sex and is related to attractiveness. Evolution and Human Behavior 28, 3, 186-192.

E. Ashby Plant, Janet Shibley Hyde, Dacher Keltner, and Patricia G. Devine. 2000. The gender stereotyping of emotions. Psychology of Women Quarterly 24, 1, 81-92.

Frank E. Pollick, Helena M. Paterson, Armin Bruderlin, and Anthony J. Sanford. 2001. Perceiving affect from arm movement. Cognition 82, 2, B51-B61.

Philippe G. Schyns, Lucy S. Petro, and Marie L. Smith. 2009. Transmission of facial expressions of emotion co-evolved with their efficient decoding in the brain: Behavioral and brain evidence. PLoS One 4, 5, e5625.

Rhoda Kesler Unger. 1979. Toward a redefinition of sex and gender. American Psychologist 34, 11, 1085-1094.

Ekaterina P. Volkova, Betty J. Mohler, Trevor J. Dodds, Joachim Tesch, and Heinrich H. Bülthoff. 2014. Emotion categorization of body expressions in narrative scenarios. Frontiers in Psychology 5, 623, 1-11.

Anna C. Wellerdiek, Markus Leyrer, Ekaterina Volkova, Dong-Seon Chang, and Betty Mohler. 2013. Recognizing your own motions on virtual avatars: Is it me or not? In Proceedings of the ACM Symposium on Applied Perception (SAP'13). 138.

Received November 2014; revised April 2015; accepted April 2015 